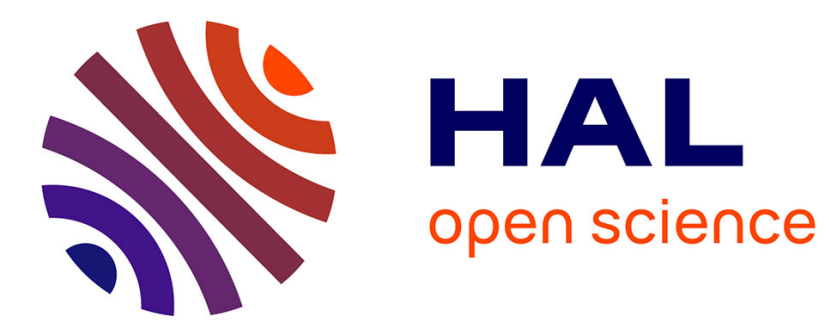

\title{
Pinning force scaling of electrospun Bi-2212 nanowire networks
}

Michael Rudolf Koblischka, Denis Gokhfeld, Crosby S Chang, Thomas Hauet, Uwe Hartmann

\section{- To cite this version:}

Michael Rudolf Koblischka, Denis Gokhfeld, Crosby S Chang, Thomas Hauet, Uwe Hartmann. Pinning force scaling of electrospun Bi-2212 nanowire networks. Solid State Communications, 2017, 264, pp.1618. 10.1016/j.ssc.2017.07.002 . hal-01710779

\section{HAL Id: hal-01710779 \\ https://hal.science/hal-01710779}

Submitted on 16 Feb 2018

HAL is a multi-disciplinary open access archive for the deposit and dissemination of scientific research documents, whether they are published or not. The documents may come from teaching and research institutions in France or abroad, or from public or private research centers.
L'archive ouverte pluridisciplinaire HAL, est destinée au dépôt et à la diffusion de documents scientifiques de niveau recherche, publiés ou non, émanant des établissements d'enseignement et de recherche français ou étrangers, des laboratoires publics ou privés. 


\title{
Pinning force scaling of electrospun Bi-2212 nanowire networks
}

\author{
Michael R. Koblischka \\ Institute of Experimental Physics, Saarland University, Campus C 6 3, 66123 Saarbrücken, Germany. \\ Denis Gokhfeld \\ Kirensky Institute of Physics, Federal Research Center KSC SB RAS, Akademgorodok 50/38, Krasnoyarsk, 660036 Russia. \\ U. Hartmann \\ Institute of Experimental Physics, Saarland University, Campus C 6 3, 66123 Saarbrücken, Germany. \\ Crosby Chang, Thomas Hauet \\ Institut Jean Lamour, UMR CNRS-Université de Lorraine, 54506 Vandœvre-lès-Nancy, France.
}

\begin{abstract}
Flux pinning forces were determined on different network samples of superconducting $\mathrm{Bi}_{2} \mathrm{Sr}_{2} \mathrm{CaCu}_{2} \mathrm{O}_{8}$ (Bi-2212) nanowires prepared by the electrospinning technique. We employed magnetization data determined by SQUID magnetometry in a wide temperature range $10 \mathrm{~K}<T<35 \mathrm{~K}$, where a strong superconducting signal prevails. The scaling analysis of the pinning forces was applied to interprete the data obtained. Both pure and Li-doped Bi2212 nanowire networks exhibit a peak position of $h_{0} \sim 0.11$, which is smaller than the expected value of $h_{0}=0.2$ indicating flux pinning at grain boundaries or extended defects. For the flowing currents through such a network, the crystallographic anisotropy and the percolation play an important role, resulting in reduced peak positions as compared to bulk samples.
\end{abstract}

Keywords:

PACS: 74.70.Es, 74.72.-h, 74.25.Ha

\section{Introduction}

Recently, superconducting nanowires of $\mathrm{Bi}_{2} \mathrm{Sr}_{2} \mathrm{CaCu}_{2} \mathrm{O}_{8}$ (Bi-2212) could successfully be prepared by employing the electrospinning technique $[1,2,3]$. The nanowires themselves were found to be polycrystalline, consisting of nanometer-sized grains of random orientation and exhibit diameters of about $250 \mathrm{~nm}$, which is comparable to the London penetration depth, $\lambda_{0}$. The networks of these nanowires formed by electrospinning and the subsequent heat treatment have an extremely low density and show numerous interconnects between the nanowires which enable a current flow through the entire network. The transport and magnetization measurements [2, 4] revealed that the currents can flow through the entire sample perimeter even at applied magnetic fields of $10 \mathrm{~T}$. These properties of the nanowire network samples are very interesting from the underlying physics and for possible applications. Therefore, in this letter, we analyze the flux pinning forces obtained from $M(H)$ measurements using the model of Dew-Hughes (DH) $[5,6]$ to gain a deeper understanding of the magnetic behavior of the nanowire networks, which may be interesting for certain applications whereever the weight of the samples counts, and we compare the resulting pinning force scaling diagrams to such of other types of $\mathrm{Bi}-2212$ and $\mathrm{Bi}-2223$ materials published in the literature.

\section{Experimental procedure}

The Bi-2212 nanowire networks were fabricated using the electrospinning technique from an aqueous solution of polyvinylpyrrolidone (PVP, $\left.M_{W} 1,300,000\right)$, bismuth(III) acetate, strontium acetate hydrate, calcium acetate hydrate and copper(II) acetate monohydrate and followed by calcination treatment of the electrospun polymer/inorganic composite fibers including one oxygenation step in pure $\mathrm{O}_{2}$. The chemicals were commercially available (Alfa Aesar GmbH \& Co KG, purity above $99.99 \%$ ) and used as received without further purification. X-ray analysis confirmed that the samples are pure Bi-2212 phase with some residing carbon. For the sample with Li doping, $30 \mathrm{x} .-\% \mathrm{Li}$ acetate was added to the precursor solution. Further details of the nanowire fabrication procedure are given elsewhere[3]. The density of our nanowire networks is found to be equal to $0.0459 \mathrm{~g} / \mathrm{cm}^{3}$, which is considerably lower than the theoretical density of bulk Bi-2212 $\left(6.4 \mathrm{~g} / \mathrm{cm}^{3}\right)$.

The magnetization of the nanowire networks was measured using a SQUID magnetometer (Quantum Design MPMS3) with $\pm 7 \mathrm{~T}$ magnetic field applied perpendicular to the sample surface. The onset of superconductivity takes place at $76 \mathrm{~K}$, which 
is somewhat lower than the bulk $T_{c}$ of the Bi-2212 phase ( 85 K) $[2,4]$.

\section{Results and discussion}

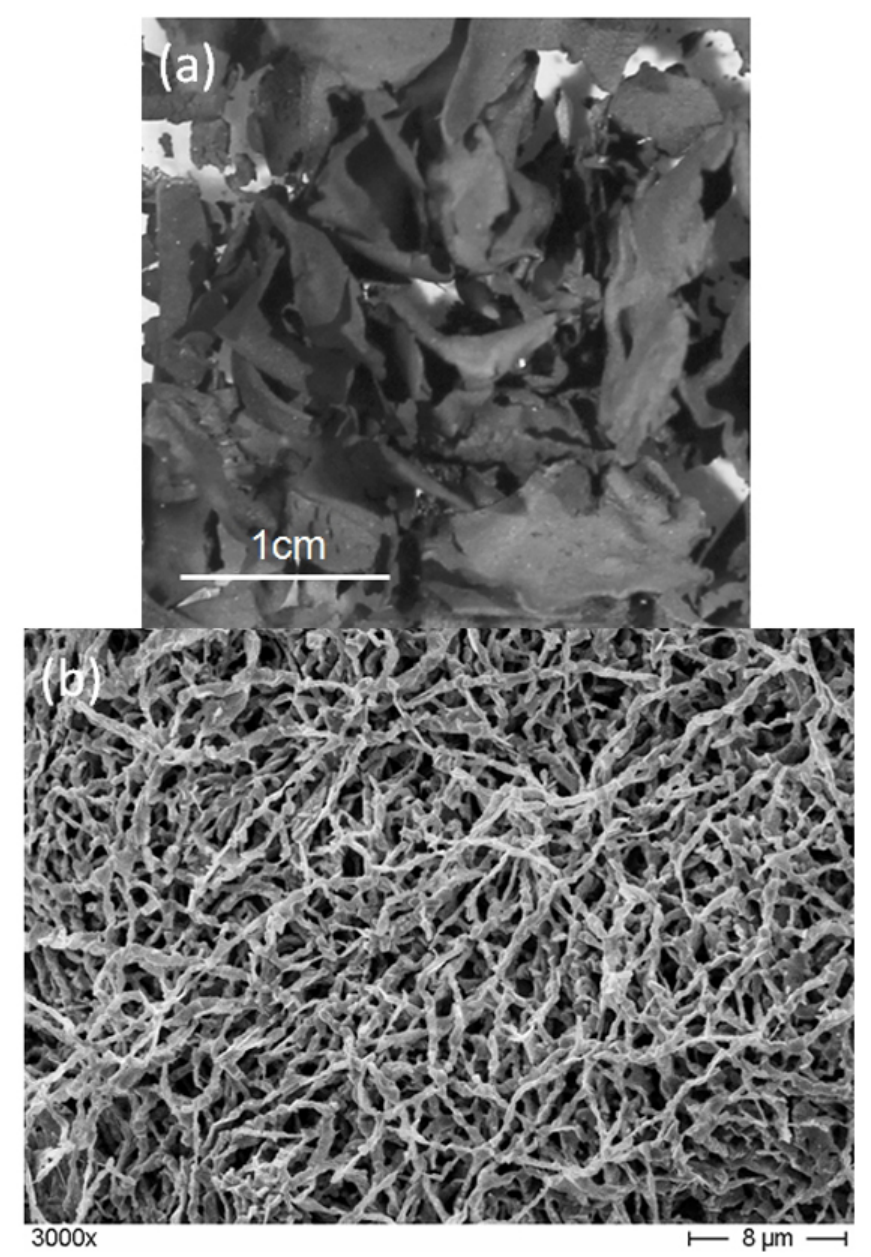

Figure 1: (a) Optical image of heat-treated nanowire network samples. (b) SEM image (magnification $3000 \times$ ) of the nanowire network after the final heat treatment.

The microstructure of the $\mathrm{Bi}-2212$ nanowire networks are presented in Figs. $1(\mathrm{a} / \mathrm{b})$ after the final heat treatment. The nanowire networks (a) look like a thin fabric and are extremely brittle. Due to the numerous interconnects between the individual nanowires as shown in (b), transport currents can flow through the entire nanowire network sample.

Figure 2 shows magnetization hysteresis loops of the Bi2212 and $\mathrm{Bi} 2212+\mathrm{Li}$ samples measured at $T=15 \mathrm{~K}$. The loops exhibit the typical asymmetric shape of polycrystalline materials [7]. Both loops show an additional magnetic signal overlaid on the superconducting loop. For pure Bi-2212, there is a diamagnetic signal, and for the Li-doped sample, the signal is paramagnetic.

The dependence of the pinning force density on the applied magnetic field was determined by $F_{p}(H)=\mu_{0} H \times J_{c}(H)$. The $J_{c}(H)$ datapoints are obtained from the width of the experimental loops using the Bean formula. In Figs. 3, 4, the normalized

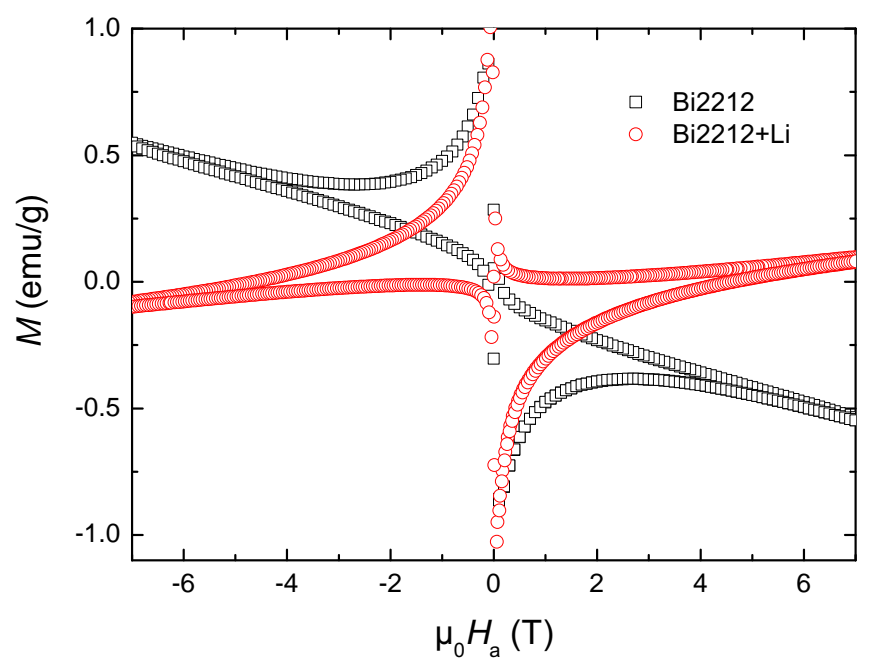

Figure 2: Magnetization hysteresis loops measured at $T=15 \mathrm{~K}$.

pinning force $f_{p}=F_{p} / F_{p 0}$ is plotted as a function of the reduced field $h=H / H_{\text {irr }}$ for different $T$, here $F_{p 0}$ is the maximal pinning force density at given $T$. For high- $T_{c}$ superconductors, the values of $H_{\text {irr }}$ are usually determined from a logarithmic plot. Here we see that noise appears in the $J_{c}(H)$ datapoints right at $H>H_{\text {irr }}$. The noise level was subtracted before the $f_{p}(h)$ dependencies were plotted. The resulting $f_{p}(h)$ dependencies at all temperatures are almost coinciding, thus the $f_{p}$ scaling is well developed in both types of samples studied here.

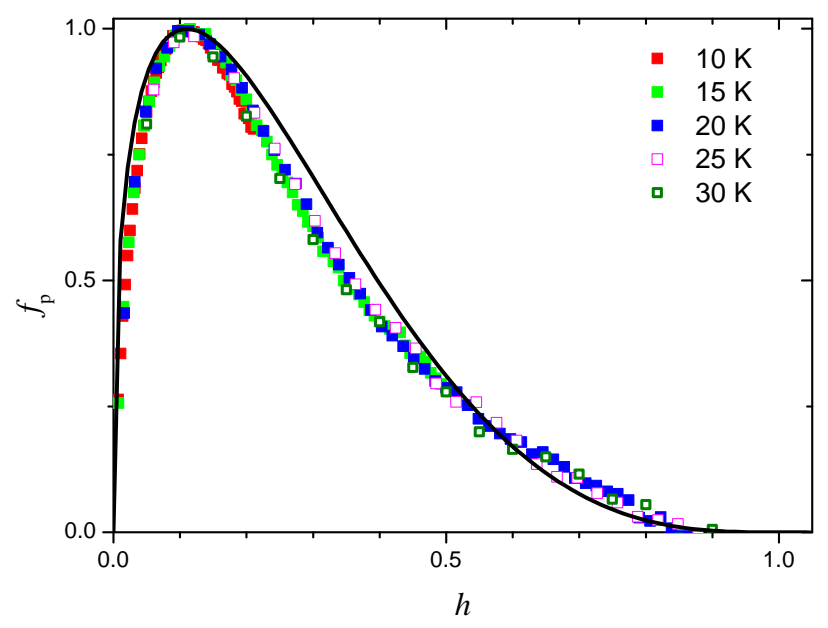

Figure 3: Scaling of the pinning forces of the Bi2212 sample. The solid line represents the ECSM fitting curve with $p=0.37$ and $q=3$.

The pinning force scaling reveals a peak position, $h_{0}$, of $\sim 0.11$. This value is smaller than $h_{0}=0.2$, which is expected for the flux pinning at $2 \mathrm{D}$ objects like grain boundaries [5] and was found for various Bi-based superconducting materials in the literature $[8,9,10,11,12,13,14]$. This small value of $h_{0}$ reflects the influence of anisotropy and percolation, which was described already in Refs. [9, 15]. The supercurrents 


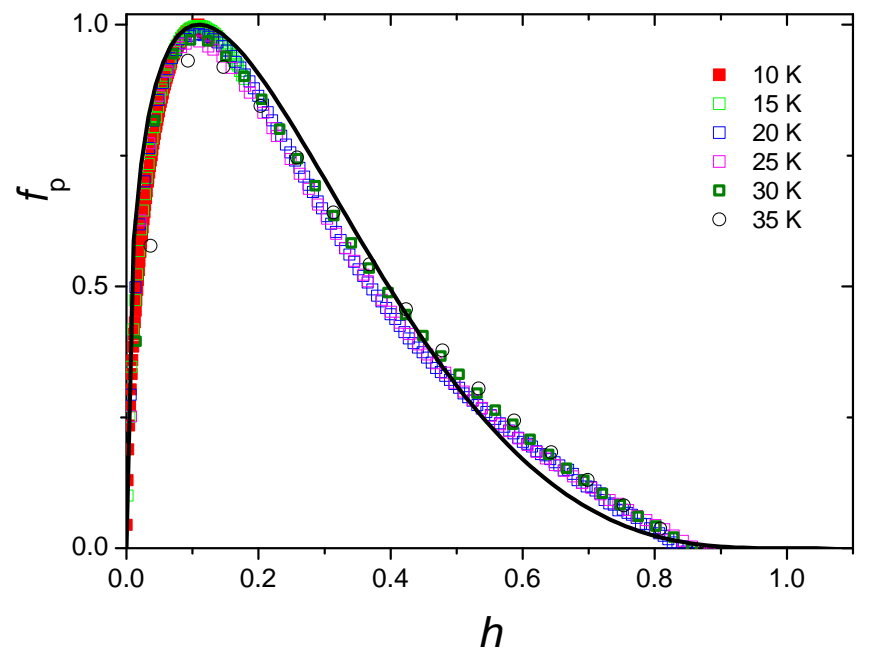

Figure 4: Scaling of the pinning forces of the Bi2212+Li sample. The solid line represents the ECSM fitting curve with $p=0.37$ and $q=3$.

flowing within an individual nanowire have to pass grains with many different orientations, so the anisotropy factor (i.e., the anisotropy of the upper critical fields) is important to describe the resulting current. According to Ref. [15] regarding the pinning force analysis in bulk $\mathrm{MgB}_{2}$ samples, an anisotropy factor larger than 4, which is certainly fulfilled for Bi-2212, yields a position of $h_{0}=0.1$ for the grain boundary pinning which otherwise would give $h_{0}=0.2$. The influence of percolation effects on the peak position was found to be much smaller as described in Ref. [15]. However, the percolation effects were also found to reduce the peak positions as compared to the $\mathrm{DH}$ model. Therefore, one can safely conclude that the peak position $h_{0} \sim 0.1$ describes grain boundary pinning in the case of an anisotropic material and percolating currents.

For a bulk type-II superconductor with sizes much higher than the London penetration depth $\lambda_{0}$, the dependence of the critical current density $J_{c}$ on the magnetic field $H$ can be expressed by:

$$
J_{c}(H)=J_{\mathrm{c} 0} \frac{1-\left|H / H_{c 2}\right|^{\alpha}}{1+\left|H /\left(h_{1} H_{c 2}\right)\right|^{\alpha}},
$$

where $\alpha \leq 1$. This expression is a modification of the KimAnderson and Urban [16] dependencies. The extended critical state model $[17,18,19]$ supports a faster decreasing of the $J_{c}(H)$ dependence due to an extension of the equilibrium surface. A type-II superconductor with size being comparable to $\lambda_{0}$ has the following $J_{c}(H)$ dependence:

$$
J_{c}(H)=J_{\mathrm{c} 0} \frac{1-\left|H / H_{c 2}\right|^{\alpha}}{1+\left|H /\left(h_{1} H_{c 2}\right)\right|^{\alpha}}\left(1-\left|H / H_{\text {irr }}\right|\right)^{n},
$$

where $n$ is the index defined by the geometry of the grain, $n=$ 3 for a cylindrical sample and $n=2$ for a thin plate[19].

We are interested in the behavior of the pinning force in the field range $H_{p}<H \ll H_{c 2}$. Given $h_{1} \ll 1$ and $H \ll H_{c 2}$, one obtains the pinning force density from eq. (2):

$$
F_{p}(H) \approx|H|^{1-\alpha}\left(1-\frac{|H|}{H_{\mathrm{irr}}}\right)^{n} J_{\mathrm{c} 0}\left(h_{1} H_{c 2}\right)^{\alpha}
$$

After substitution for $\alpha=1-p$ and $n=q$, the well-known scaling law of Dew-Hughes [5] is resulted:

$$
f_{p}(h)=\left(\frac{h}{h_{0}}\right)^{p}\left(\frac{1-h}{1-h_{0}}\right)^{q}
$$

where $h_{0}$ is the position of the maximum, $h_{0}=p /(p+q)$. The position of the pinning force maximum is easily determined from graphs such that one can find the value of $\alpha$ from $\alpha=1-n /\left(1 / h_{0}-1\right)$. Given $h_{0}=0.11$ and $n=3$ we obtain $\alpha=0.63$. This corresponds to $p=0.37$ and $q=3$. These values differ from each other and are typical for anisotropic and polycrystalline superconductors [9, 15]. The solid line in Figs. 3, 4 represents the $f_{p}(h)$ dependence computed using eq. (3) and gives a good fit to the data in both cases.

Both types of nanowire networks (undoped and doped with Li) were found to show a good scaling of the flux pinning force data, and the peak, $h_{0}$ is located at $\sim 0.11$, which is clearly smaller than the expected value of 0.2 as found for Bi-based superconductors $[8,9,10,11,12,13,14]$.

Here it should be noted that the high- $h$ region of the $F_{p^{-}}$ scaling plots is influenced by several factors, (i) the magnetometer shows noise being close to its resolution, and (ii) the currents at high fields are very small and highly percolative. As a consequence, the irreversibility field is hard to determine from the plots. Nevertheless, the obtained scalung of the flux pinning forces turned out to be quite good in the temperature range investigated here, and the peak position of the $f_{p}$-plot is a good indicator of the active pinning mechanism, as flux creep effects do not play a role here.

It is interesting to note that the influence of anisotropy and percolation were only recently introduced to the pinning force analysis [15], when regarding the behavior of bulk, polycrystalline $\mathrm{MgB}_{2}$ samples. In superconducting samples with a forced texture (bulk samples or tapes), the anisotropy does only play a minor role, and only recently, bulk, polycrystalline FeSe samples $[20,21]$ were found to behave similar to polycrystalline $\mathrm{MgB}_{2}$ due to a well-developed coupling between the grains. According to Ref. [15], the changes in $F_{p}$ due to changes in the percolation threshold are comparatively small since $p_{c}$ is expected to vary only between 0.2 and 0.3 in three-dimensional systems [22]. The percolation threshold mainly depends on the number of connections between the grains; thus, clean dense samples have a comparatively small $p_{c}$ and porous or dirty samples with a large amount of secondary phases have a larger $p_{c}$. For the present nanowire networks, the character of an individual nanowire is more 2D, as only a small number of grains form the entire nanowire. This number can be as small as 3-5 grains. Furthermore, the residing carbon in the nanowire samples may be located at the grain boundaries, and this effect is even enhanced in the case of the Li-doped samples, where we assume that $\mathrm{Li}$ is also found in the grain boundaries [23, 24]. Therefore, the nanowire network samples with their high porosity and the dirty grain boundaries should exhibit a stronger influence of $p_{c}$ as compared to $\mathrm{MgB}_{2}$ bulks analyzed in Ref. [15], which causes a move of the peak position in the $f_{p}(h)$-diagram to lower values as observed here. 


\section{Conclusions}

We have performed a pinning force scaling on the data obtained from electrospun $\mathrm{Bi}-2212$ nanowire networks based on magnetization data. The analysis was performed using the Bean model and the ECSM model. The obtained scaling was found to quite good in the investigated temperature range $10 \mathrm{~K}<T<$ $35 \mathrm{~K}$, and the peak position of the $f_{p}$-plots were found at $\sim 0.11$. Due to anisotropy and percolation, this value is reduced as compared to the value obtained for the grain boundary pinning in bulk materials.

This work is supported by the DFG project Ko2323/8, which is gratefully acknowledged.

[1] E. A. Duarte, P. A. Quintero, M. W. Meisel, J. C. Nino, Physica C 485 (2013) 109-113.

[2] M. R. Koblischka, X. L. Zeng, T. Karwoth, T. Hauet, U. Hartmann, AIP Advances 6 (2016) 035115.

[3] X. L. Zeng, M. R Koblischka, T. Karwoth, T. Hauet, U. Hartmann, Supercond. Sci. Technol. 30 (2017) 035014.

[4] M. R. Koblischka, X. L. Zeng, T. Karwoth, T. Hauet, U. Hartmann, IEEE Trans. Appl. Supercond. 26 (2016) 1800605.

[5] D. Dew-Hughes, Philos. Mag. 30 (1974) 293-305.

[6] M. R. Koblischka, A.J.J. van Dalen, T. Higuchi, S. I. Yoo, M. Murakami, Phys. Rev. B 58 (1998) 2863-2867.

[7] S. Senoussi, J. Phys. III France 2 (1992) 1041-1257.

[8] G. Yang, P. Shang, S. D. Sutton, I. P. Jones, J. S. Abell, C. E. Gough, Phys. Rev. B 48 (1993) 40544060.

[9] P. Fabbricatore, C. Priano, A. Sciutti, G. Gemme, R. Musenich, R. Parodi, F. Gömöry, J. R. Thompson, Phys. Rev. B 54 (1996) 12543-12550.

[10] M. R. Koblischka, Physica C 282-287 2193-2194 (1997).

[11] S. Chu, M. E. McHenry, IEEE Trans. Appl. Supercond. 7 (1999) 11501153.

[12] H. Sasakura, O. Miura, D. Ito, IEEE Trans. Appl. Supercond. 9 (1999) 2332-2335.

[13] B. Ni, M. Kikuchi, E. S. Otabe, IEEE Trans. Appl. Supercond. 13 (2003) 3695-3698.

[14] M. R. Koblischka, J. Sosnowski, Eur. Phys. J. B 44 (2005) 277-280.

[15] M. Eisterer, Phys. Rev. B 77 (2008) 144524.

[16] E. W. Urban, J. Appl. Phys. 42 (1971) 115-127.

[17] D. M. Gokhfeld, D. A. Balaev, M. I. Petrov, S. I. Popkov, K. A. Shaykhutdinov, V. V. Val'kov, J. Appl. Phys. 109 (2011) 033904.

[18] D. M. Gokhfeld, Phys. Solid State 56 (2014) 2380-2386.

[19] D. M. Gokhfeld, J. Phys. Conf. Ser. 695 (2016) 012008.

[20] A. Leo, G. Grimaldi, A. Guarino, F. Avitabile, A. Nigro, A. Galluzzi, D. Mancusi, M. Polichetti, S. Pace, K. Buchkov, E. Nazarova, S. Kawale, E. Bellingeri, C. Ferdeghini, Supercond. Sci. Technol. 28 (2015) 125001.

[21] M. R. Koblischka, M. Muralidhar, Int. J. Mod. Phys. B 30 (2016) 1630017.

[22] S. C. van der Marck, Phys. Rev. E 55 (1997) 1514.

[23] I. Parinov, Microstructure and properties of high- $T_{c}$ superconductors, 2nd edition (Springer: Berlin, Heidelberg, 2012) p. 283.

[24] T. B. Tang, C. R. Li, Chin. J. Phys. 36 (1998) 280-287. 